\title{
The three-state Potts model on a triangular lattice
}

\author{
Hyunggyu Park \\ Department of Physics \\ Inha University \\ Inchon, 402-751, Korea
}

\begin{abstract}
We study the phase diagram of the three-state Potts model on a triangular lattice with general interactions (ferro/antiferromagnetic) between nearest neighbor spins. When the interactions along two lattice-vector directions are antiferromagnetic and infinitely strong, this model becomes equivalent to a six-vertex model and exhibits a first-order (KDP) transition from an ordered phase into a critical phase. Comparing the excitations occurred by relaxing the restriction of infinite-strength interactions and those in the eight-vertex model, we analytically obtain the critical index for those excitations and demonstrate the existence of a critical phase for the case of finite antiferromagnetic interactions in two directions and ferromagnetic interactions in the other direction. When the interactions are antiferromagnetic in all three directions, Monte Carlo simulations show that a first-order line emerges from the KDP point and separates completely an ordered phase and a disordered phase. Along the special line where all three antiferromagnetic interactions have the same strength, the cell-spin analysis reveals that the symmetry of the ground states is dual to the symmetry of the $n=3$ ferromagnetic cubic model which is known to exhibit a first-order phase transition.
\end{abstract}

\section{Introduction}

The ferromagnetic three-state Potts model has been studied extensively. In two dimensions, its critical properties which are independent of underlying lattices are known exactly by the extended scaling[1], 2] and/or the conformal invariance[3]. When interactions between neighboring spins become antiferromagnetic, the critical properties vary with the structure of underlying lattices. The symmetry of the antiferromagnetic ground states is constrained 
by the structure of the underlying lattice. For example, the antiferromagnetic three-state Potts model on a square lattice is disordered at all temperatures, but on a triangular lattice a first-order phase transition appears at a finite temperature. By adding ferromagnetic next-nearest-neighbor interactions, the antiferromagnetic three-state Potts model on a square lattice exhibits a sequence of two Kosterlitz-Thouless (KT) transitions [4]. This model possesses a ground-state symmetry similar to the ferromagnetic sixstate clock model[5]. In the case of mixed-type interactions, i.e. ferromagnetic in one direction and antiferromagnetic in the other direction, Monte Carlo simulations [6] and transfer matrix calculations [7] on a square lattice indicate that there is a KT-like infinite-order phase transition from a massless lowtemperature phase into a disordered phase with an essential singularity at a finite temperature. In this paper we examine the phase diagram of the three-state Potts model with antiferromagnetic and mixed-type interactions on a triangular lattice.

The three-state Potts model on a square lattice can be mapped to a 27vertex model on its dual lattice[1] (section 2). The triangular-lattice model with nearest-neighbor interactions can be viewed as the square-lattice model with nearest- and next-nearest-neighbor interactions by distorting the lattice properly. We map this model onto a 27 -vertex model on its dual lattice. When the interactions along two lattice-vector directions are antiferromagnetic and infinitely strong, only six vertex configurations survive. From the exact solution of the six-vertex model[8], we find a first-order (KDP) transition from an ordered phase into a critical phase. By the stability analysis similar to the work by den Nijs et al [4] for the square-lattice model, we demonstrate the existence of a critical phase for the case of finite antiferromagnetic interactions in two directions and ferromagnetic interactions in the other direction. It implies that there is a KT-type transition from a critical phase into a disordered phase in the case of mixed-type interactions.

The three-state Potts model with the isotropic antiferromagnetic interactions has been studied previously by the real-space renormalization 9], series expansions [10], and Monte Carlo simulations [11]. It has been shown to exhibit a strong first-order transition. In section 3, its ground-state symmetry is investigated. We construct the cell-spin hamiltonian by calculating domain wall energies and show that this model can be renormalized to the dual model of the $n=3$ cubic model[12]. The calculated values of coupling constants of this cubic model guarantee that the phase transition of our isotropic model 
is of first order.

When the interactions are anisotropic, the chirality in domain wall energies appear. In section 4, we perform Monte Carlo simulations to understand the role of the chirality in this model. We find that a first-order line emerges from the KDP point and separates completely the antiferromagnetic ordered phase and the disordered phase. The chirality neither changes the nature of the phase transition, nor gives rise to any other phase transition in the antiferromagnetic region. Pleliminary results for the antiferromagnetic case have been published separately elsewhere[13].

We conclude in section 5 with a brief summary.

\section{Mapping to the vertex model and stability analysis}

Consider the three-state Potts model on a square lattice with nearest-neighbor and next-nearest-neighbor interactions. The hamiltonian of the model, $H$, is given as

$$
-H=\sum_{<i, j>} K \delta_{\sigma_{i} \sigma_{j}}+\sum_{(i, j)^{\prime}} L_{1} \delta_{\sigma_{i} \sigma_{j}}+\sum_{(i, j)^{\prime \prime}} L_{2} \delta_{\sigma_{i} \sigma_{j}}
$$

where $\langle.$.$\rangle and (.$.$) denote nearest neighbors and next-nearest neighbors$ respectively (see Fig.1). $\sigma_{i}$ is the Potts spin at site $i$ which takes the values of $0,1,2$ and $\delta$ is the Kronecker delta function. The three-state Potts model on a triangular lattice with nearest-neighbor interactions only can be obtained by taking either $L_{1}$ or $L_{2}$ to be zero. Here we take $L_{2}=0$.

A three-to-one mapping to a 27 -vertex model on the dual lattice is obtained by assigning arrows or zeros to the bonds of the dual lattice. As one goes around a dual lattice site clockwise, an outgoing (incoming) arrow is assigned on the encountered bond if the value of the Potts spin is increased (decreased) by one with modulo 3 going across the bond. If the value of the Potts spin is unchanged, we assign a zero on the encountered bond (see Fig.1). Bolzmann weights in terms of $u \equiv \exp (K)$ and $v \equiv \exp \left(L_{1}\right)$ and the number of vertices are shown in Fig.2. In the limit $K \rightarrow-\infty(u=0)$, only six vertices are left with nonvanishing Boltzmann weights. By normalizing Boltzmann weights with respect to the last pair of unpolarized vertices, we find $a=1 / v$ and $b=1$ where $a$ and $b$ are the Boltzmann weights of the first 
two pairs of vertices. The single parameter $\Delta$ of the six-vertex model [8, 14] is given as

$$
\Delta=\frac{1}{2}\left(\frac{a}{b}+\frac{b}{a}-\frac{1}{a b}\right)=\frac{1}{2 v} .
$$

From the exact solution of the six-vertex model 8, 14], we find that there is a first-order KDP transition at $\Delta=1(v=1 / 2)$ from an antiferromagnetic ordered phase $(v<1 / 2)$ into a critical phase $(v>1 / 2)$ (see Fig.3).

With finite $K(u>0)$, the vertices with zeros on the bonds can appear. Following the analysis of den Nijs et al [4] for the antiferromagnetic threestate Potts model on a square lattice, the most important vortex excitations which drive the critical phase into the disordered phase are the bound pairs of vortex states (Figs.2(e) and $2(f)$ ) which have vorticity of \pm 6 (Fig.4). The scaling dimension of these excitations can be obtained by using the well-known relation between scaling dimensions for excitations of different vorticities such as 15

$$
x_{m}=\left(\frac{m}{n}\right)^{2} x_{n}
$$

where $x_{m}$ is the scaling dimension for excitations of vorticity $m$ and zero spinwave excitation index. The scaling dimension $x_{4}$ is known exactly from the Baxter's solution of the eight-vertex model[16]. Thus the scaling dimension for excitations of vorticity 6 is given as

$$
x_{6}=\left(\frac{9}{4}\right) x_{8 V}
$$

where $x_{8 V}=2-y_{8 V}=2-\frac{2}{\pi} \cos ^{-1}(-\Delta)$. The critical exponent $y_{6}=2-$ $x_{6}$ becomes negative when $v>v^{*}=1 /\left(2 \sin \left(\frac{\pi}{18}\right)\right)$. So these excitations are irrelevant in this region and the critical phase persists for a small but finite value of $u$. For $v<v^{*}$, they are relevant with respect to the $u=$ 0 line. In this region, these two bound pairs can be dissociated and the system becomes disordered for any finite value of $u$. Thus we can draw the phase diagram for small $u$ in the axis of $v$ (see Fig.5). As $v$ increases from the antiferromagnetically ordered phase, a first-order transition into the disordered phase is expected near $v \simeq 0.5$ and subsequently a continuous KT-type transition into the critical phase near $v \simeq 2.879$. Notice that the bound pairs of vortex states in Fig. 4 are always confined for small $u$ because a string of zeros are generated by pulling the bound pairs apart. 


\section{Ground state symmetry and cell-spin hamil- tonians}

First we study the ground state symmetry of the three-state Potts model with antiferromagnetic nearest-neighbor interactions and ferromagnetic nextnearest-neighbor interactions on a square lattice 4 , 5]. Its hamiltonian is given in eq.(1) with $K<0$ and $L=L_{1}=L_{2}>0$. This model has six equivalent ground states. The unit cells of these ground states are shown in Fig.6. At zero temperature, the system becomes a periodic array of one of these unit cells. As the temperature goes higher, the system becomes a mixture of these unit cells and the domain walls between different unit cells appear. The excitation energies per unit length for these domain walls, when they are straight and do not meander, are easy to determine

$$
E_{i, i+1}=L, \quad E_{i, i+2}=2 L-K / 2, \quad \text { and } \quad E_{i, i+2}=2 L-K,
$$

where $i$ is an integer of modulo 6 and $E_{i j}$ is the excitation energy of the domain wall between two ground states, $i$ and $j$. There is no chirality in domain wall energies; $E_{i j}=E_{j i}$. We observe from eq.(5) that the domain wall energy $E_{i j}$ depends on $|i-j|$ only and is a periodic function of $i$ and $j$ with periodicity of 6 . This is a symmetry of the six-state clock model where the domain wall energies depend only on the angle between states (Fig.7). If we assign a cell spin $\sigma(\sigma=1, \cdots, 6)$ for each unit cell, the cellspin hamiltonian reduces to the ordinary six-state ferromagnetic clock model hamiltonian

$$
-H=-\sum_{<i, j>} J\left[1-\cos \frac{2 \pi}{6}\left(\sigma_{i}-\sigma_{j}\right)\right],
$$

with $L=-K / 2=J / 2$. The six-state clock model is known to exhibit a sequence of two KT transitions 17. This explains why there exists a critical fan in the antiferromagnetic three-state Potts model on a square lattice.

Now we consider the three-state Potts model with antiferromagnetic nearest-neighbor interactions on a triangular lattice. The coupling constants along three different lattice-vector directions are denoted by $K_{i}(i=1,2,3)$. All $K_{i}$ 's are negative. Similar to the above square-lattice model, there are six equivalent ground states; three up-states $U_{i}$ and three down-states $D_{i}$ $(i=1,2,3)$. Their unit cells are shown in Fig.8. We say that the up-states have a positive helicity and the down-states a negative helicity. Domain wall 
energies between these ground states depend on their directions and also on the chirality. Consider the domain wall between two up-states $U_{1}$ and $U_{2}$ in the direction 1 (Fig.9). When $U_{1}$ is left and $U_{2}$ is right to the domain wall, the excitation energy per unit length of this domain wall is $-K_{3}$. If the ground states are interchanged, the domain wall energy becomes $-K_{2}$. So when $K_{2} \neq K_{3}$, there is a chirality in the domain wall energies. In general, one can find that the domain wall energy between two up-states $U_{i}$ and $U_{j}$ in the direction 1 is $E_{1}=-K_{2} \delta_{i-1, j}-K_{3} \delta_{i+1, j}$. When $i=j-1(j+1)$, we call that the domain wall has a positive (negative) chirality. Similarly, the domain wall energies between two up-states in the other directions can be obtained easily and the result is

$$
E_{i}^{ \pm}(U U)=-K_{i \mp 1},
$$

where the subscript $i$ denotes the direction of the domain wall, the superscript \pm the chirality, and $U U$ in the parenthesis represents the domain wall energy between up-states. Repeating the same analysis on the domain walls between down-states and also between up- and down-states, we find

$$
\begin{aligned}
& E_{i}^{ \pm}(D D)=-K_{i \pm 1}, \\
& E_{i}^{ \pm}(U D)=-\frac{1}{3}\left(K_{i+1}+K_{i-1}\right) .
\end{aligned}
$$

Notice that the domain wall energies between up- and down-states do not depend on the chirality.

The symmetry structure of the six ground states is drawn in Fig.10. There is a ferromagnetic chiral (or helical) three-state Potts model symmetry [18] in each triangle. And these triangles are linked by the symmetry of a ferromagnetic nonchiral Ising model. When $K_{1}=K_{2}=K_{3}$ (isotropic case), the chirality disappears in both triangles. Even though the number of ground states is the same as in the square-lattice model discussed previously, the symmetry between the ground states is completely different from each other.

First consider the $K_{1}=K_{2}=-\infty(u=0)$ limit. This is the six-vertex model limit (see section 2). In this limit, only four walls can survive and their energies are

$$
E_{1}^{+}(U U)=E_{2}^{-}(U U)=E_{1}^{-}(D D)=E_{2}^{+}(D D)=-K_{3} .
$$

Domain walls in the direction 3 are not allowed and the up-states and downstates cannot coexist. There will be no isolated loop excitations of domain 
walls because there exist no pairs of domain walls in the same direction with different chirality. Thus any excitation of this model can be represented by the zig-zag lines of the domain walls of types $E_{1}^{+}(U U)$ and $E_{2}^{-}(U U)$, or $E_{1}^{-}(D D)$ and $E_{2}^{+}(D D)$ (Fig.11). The domain wall energy is given by $v=\exp \left(K_{3}\right)$ and there is no energy cost at the crossing of the walls. By mapping to the vertex model with Bolztmann factors normalized with respect to the last pairs of the vertices, one can recover the six-vertex model with $a=1 / v$ and $b=1$ as expected (Fig.12). So in the $u=0$ limit, the cell-spin approach produces the exact result.

For the isotropic model $(u=v=\exp (K))$, there is no chirality. Assign two types of cell spins, $t$ and $s(t=1,2,3$ and $s=1,2)$, for each unit cell. The $s=1$ state represents the up-states and the $s=2$ state the down-states. Each of three states inside the up- or down-states is represented by the spin $t$. Then the cell-spin hamiltonian can be written as

$$
-H=\sum_{<i, j>}\left[-K \delta_{s_{i} s_{j}} \delta_{t_{i} t_{j}}+\frac{K}{3} \delta_{s_{i} s_{j}}+\frac{2}{3} K\right],
$$

which is exactly the same as the hamiltonian of the so-called $\left(q_{s}, q_{t}\right)$ model 19 with $q_{s}=2$ and $q_{t}=3$. For $q_{t}=2$ it is known as the cubic model 12]. Nature of phase transitions does not depend on the underlying lattice structure for ferromagnetic models like the above cell-spin model $(-K>0)$. So we study the above model on a square lattice which has been investigated in details. The duality relation between the $\left(q_{s}, q_{t}\right)$ model and the $\left(q_{t}, q_{s}\right)$ model is known on a square lattice 19]. After dropping the constant term in eq.(10), one can find the dual hamiltonian

$$
-H_{D}=\sum_{<i, j>}\left[D \delta_{t_{i} t_{j}} \delta_{s_{i} s_{j}}+J \delta_{t_{i} t_{j}}\right]
$$

where

$$
\begin{aligned}
\exp (D) & =1+\frac{6}{\exp (-2 K / 3)+2 \exp (K / 3)-3} \\
\exp (J) & =1+\frac{3[\exp (K / 3)-1]}{\exp (K / 3)[\exp (-K)-1]}
\end{aligned}
$$

This is the $n=3$ cubic model hamiltonian which is known to exhibit a firstorder phase transition for $J+D / 2>0$ and a continuous phase transition 
otherwise[12]. For our model, we can prove from eq.(12) that $D>0$ and $J+D / 2>0$ for any value of $K$. Therefore the cell-spin analysis for the antiferromagnetic isotropic three-state Potts model on a triangular lattice shows that there must be a first-order transition rather than a continuous transition and the symmetry of the ground states is dual to the symmetry of the $n=3$ cubic model. Nature of the transition is consistent with the Monte Carlo results[1]].

On the other side of the phase diagram $(K<0, L>0)$, there exists a critical phase. There are infinitely many ground states in the thermodynamic limit. In the ground states, Potts spins are ordered completely along the direction 3 and nearest-neighbor spins in the other directions should be different (Fig.13). This model may exhibit the same critical behavior as the squarelattice model with mixed-type nearest-neighbor interactions only; antiferromagnetic in one direction and ferromagnetic in the other direction [6, 0, 20]. The number of ground states grows exponentially with the linear system size $N ; n_{G}=2^{N}+2(-1)^{N}$ which is much smaller than the square-lattice model with antiferromagnetic interactions in both directions. So this model may not be disordered at all temperatures in contrast to the square-lattice anitiferromagnetic model. Our stability analysis in the previous section suggests that the transition into the critical phase is of KT type, which is consistent with numerical results by Monte Carlo simulations [6] and transfer matrix calculations [7] for the square-lattice model with mixed-type interactions.

\section{Monte Carlo simulations and phase dia- gram}

Consider the antiferromagnetic region of the phase diagram $(0<u, v<1)$. Along the $u=v$ line (isotropic case), it is shown in the previous section that the antiferromagnetic three-state Potts model on a triangular lattice can be renormalized, by the cell-spin approximation, to the dual model of the $n=3$ cubic model and then should exhibit a first-order phase transition.

For the anisotropic model, the chirality between ground states appear. There has been some interests in the role of the chirality in the ordinary ferromagnetic Potts models [18, 21]. Even though the chiral operator is relevant for the three-state Potts model, it does not introduce a new independent ex- 
ponent, i.e. $x_{C H}=x_{T}+1$ where $x_{C H}$ and $x_{T}$ are the chiral and temperature scaling dimension respectively 22. So it is believed that the scaling behavior does not change in the presence of the chirality for the three-state Potts model. This has been shown analytically for the hard hexagon model 23] and numerically for the chiral three-state Potts model [18] and the triangular Ising lattice gas [24]. The ground-state structure of our anisotropic model is much more complicated than that of the chiral three-state models. As explained in the previous section, it has two chiral three-state model symmetries linked by the Ising symmetry. So it may be quite interesting to find out what kind of role the chirality plays in this model. We use Monte Carlo simulations to investigate the phase diagram of this model.

We run conventional heat-bath Monte Carlo simulations on a $60 \times 60$ triangular lattice along the $u=v, u^{2}=v, u=v^{2}$, and $u^{3}=v$ lines. Typically a few $10^{4}$ Monte Carlo steps per spin (MCS) are performed for a given value of $u$ and $v$. We measure the antiferromagnetic order parameter $m_{A F}$ 11] and the energy density $e$. The order parameter $m_{A F}$ is defined as

$$
m_{A F}=\left\langle\frac{3}{2} \sum_{\alpha \neq \beta \neq \gamma}\left(\frac{N_{\alpha}^{A}+N_{\beta}^{B}+N_{\gamma}^{C}}{N_{t}}-\frac{1}{3}\right)^{2}\right\rangle^{\frac{1}{2}}
$$

where $N_{\alpha}^{X}$ is the number of spins of state $\alpha$ in the sublattice $X$ and $N_{t}$ is the total number of spins. Unfortunately, along all four lines, we find a very strong first-order phase transition from an antiferromagnetically ordered phase into a disordered phase. In Table I, we list the values of the coupling constant $u$ at the first-order transitions and the jump of the order parameter and the energy density. The five first-order transition points (including the KDP point) can be connected by a smooth line which starts from the KDP point $(u, v)=\left(0, \frac{1}{2}\right)$ and apparently ends at the point $(0.28,0)$ (Fig.14). As the anisotropy increases, the magnitude of the order-parameter jump becomes bigger. Our results imply that the strong first-order transition due to the cubic nature of this model preempts all possible continuous transitions in the whole antiferromagnetic region.

We also run Monte Carlo simulations in the region of mixed-type interactions where a critical phase is expected. The specific heat is measured along the $u=1 / v$ line. We find a characteristic of the KT transition in the shape of the specific heat, which has a very broad bump well outside of the expected critical phase. This is consistent with our analytical result which suggests 
the existence of KT transitions in section 2 and also with some numerical results for the square-lattice model with mixed-type interactions [6, 0, 20].

\section{Summary}

We investigate the phase diagram of the three-state Potts model on a triangular lattice with ferro/antiferromagnetic nearest neighbor interactions. In the limit of infinite antiferromagnetic interactions along two lattice-vector directions, this model maps onto the six-vertex model and we find the KDP first-order transition from an antiferromagnetic ordered phase into a critical phase. By the stability analysis, we demonstrate the existence of the KT transition from a critical phase into a disordered phase for the case of finite antiferromagnetic interactions in two directions and ferromagnetic interactions in the other direction. The critical parameter in the limit of infinite antiferromagnetic interactions is analytically obtained. In this case the ground states are equivalent to those of the three-state Potts model on a square lattice with mixed-type interactions. Our result implies that this square-lattice model should have the same kind of the KT transition, which confirms previous numerical results 20.

When the interactions are antiferromagnetic in all three directions, we find six ground states. These ground states have two chiral three-state model symmetries linked by the Ising symmetry. For the isotropic model, the chirality disappears and the cell-spin analysis reveals that the isotropic model can be renormalized to the dual model of the $n=3$ ferromagnetic cubic model with coupling constants guaranteeing the first-order phase transition. Monte Carlo simulations for the anisotropic model shows that a first-order line emerges from the KDP point and separates completely the antiferromagnetic ordered phase and the disordered phase, which indicates that the chirality is not relevant in this model.

\section{Acknowledgements}

This work was initiated when the author was at the University of Washington. He thanks M. den Nijs and T. T. Truong for useful discussions and T. C. Chey for his kind support. This work is supported in part by the Korean Science and Engineering Foundation through the SRC program of SNU-CTP 
and also through the Center for Thermal and Statistical Physics at Korea University.

\section{References}

[1] M. den Nijs, J. Phys. A 12, 1857 (1979); Phys. Rev. B 23, 6111 (1981); ibid. 27, 1674 (1983).

[2] B. Nienhuis, J. Phys. A 5, 199 (1982); in Phase Transitions and Critical Phenomena, edited by C. Domb and J. L. Lebowitz (Academic, London, 1987), Vol.11.

[3] A. A. Belavin, A. M. Polyakov, and A. B. Zamolodchikov J. Stat. Phys. 34, 763 (1984); D. Friedan, Z. Qiu, and S. Shenker Phys. Rev. Lett. 52, 1575 (1984); for a review, see J. L. Cardy, in Phase Transitions and Critical Phenomena, edited by C. Domb and J. L. Lebowitz (Academic, London, 1987), Vol.11.

[4] M. den Nijs, M. P. Nightingale, and M. Schick, Phys. Rev. B 26, 2490 (1982).

[5] J. L. Cardy, Phys. Rev. B 24, 5128 (1981).

[6] W. Kinzel, W. Selke, and F. Y. Wu, J. Phys. A 14, L399 (1981).

[7] H. J. Herrmann and H. O. Martin, J. Phys. A 17, 657 (1984).

[8] E. H. Lieb, Phys. Rev. Lett. 18, 1046 (1967); E. H. Lieb and F. Y. Wu, in Phase Transitions and Critical Phenomena, edited by C. Domb and M. S. Green (Academic, New York, 1972), Vol.1.

[9] M. Schick and R. B. Griffiths, J. Phys. A 10, 2123 (1977).

[10] I. G. Enting and F. Y. Wu, J. Stat. Phys. 28, 351 (1982).

[11] G. S. Grest, J. Phys. A 14, L217 (1981); Y. Saito, ibid. 15, 1885 (1982).

[12] D. Kim, P. M. Levy, and L. F. Uffer, Phys. Rev. B 12, 989 (1975); D. Kim and P. M. Levy, ibid. 12, 5105 (1975); D. Kim, P. M. Levy, and J. J. Sudano, ibid. 13, 2054 (1976). 
[13] H. Park and T. C. Chey, J. Korean Phys. Soc. (in press).

[14] R. J. Baxter, Exactly Solved Models in Statistical Mechanics (Academic, London, 1982).

[15] L. P. Kadanoff and A. C. Brown, Ann. Phys. (N.Y.) 121, 318 (1979).

[16] R. J. Baxter, Ann. Phys. (N.Y.) 70, 193 (1972).

[17] J. José, L. P. Kadanoff, S. Kirkpatrick, and D. R. Nelson, Phys. Rev. B 16, 1217 (1977); S. Elitzur, R. B. Pearson, and J. Shigemitsu, Phys. Rev. D 19, 3698 (1979).

[18] S. Ostlund Phys. Rev. B 24, 398 (1981); S. Howes ibid. 27, 1762 (1983); for a review, see M. den Nijs, in Phase Transitions and Critical Phenomena, edited by C. Domb and J. L. Lebowitz (Academic, London, 1988), Vol.12.

[19] M. den Nijs, Physica A 95, 449 (1979).

[20] K. Yasumura, J. Phys. A 20, 4975 (1987); M. C. Marques, ibid. 21, 1061 (1988).

[21] D. A. Huse and M. E. Fisher, Phys. Rev. Lett. 49, 793 (1982).

[22] M. den Nijs, J. Phys. A 17, L295 (1984).

[23] R. J. Baxter, J. Phys. A 13, L61 (1980).

[24] W. Kinzel and M. Schick, Phys. Rev. B 23, 3435 (1981); D. P. Landau, ibid. 27, 5604 (1983); H. Park, J. Phys. A 23, 1789 (1990); J. D. Noh and D. Kim, Int. J. Mod. Phys. B 6, 2913 (1992). 


\section{Table Caption}

Table 1 : Numerical values of the coupling constant $u_{t}$, the order-parameter jump $\Delta m_{A F}$, and the energy-density jump $\Delta e$ at the first-order transitions. Numbers in parentheses represent the errors in the last digits.

\begin{tabular}{|c||c|c|c|c|}
\hline & $u=v$ & $u^{2}=v$ & $u=v^{2}$ & $u^{3}=v$ \\
\hline \hline$u_{t}$ & $0.205(1)$ & $0.263(2)$ & $0.120(2)$ & $0.278(3)$ \\
\hline$\Delta m_{A F}$ & $0.70(3)$ & $0.81(3)$ & $0.78(2)$ & $0.88(2)$ \\
\hline$\Delta e$ & $0.17(2)$ & $0.20(2)$ & $0.21(1)$ & $0.24(1)$ \\
\hline
\end{tabular}




\section{Figure Captions}

Fig.1 : A typical configuration of Potts spin on a square lattice (thick line) and its corresponding vertex configuration on its dual lattice (thin line). Coupling constants of nearest-neighbor $(K)$ and next-nearest-neighbor interactions $\left(L_{1}, L_{2}\right)$ between Potts spins are shown.

Fig.2 : The 27-vertex respresentation on a square lattice for the three-state Potts model on a triangular lattice: vertex types, their Boltzmann factors, and the number of each vertex type.

Fig.3 : Phase diagram of the six-vertex model. The dashed line corresponds to our model at $u=0$.

Fig.4 : Bound pairs of vortex states in Figs.2(e) and $2(f)$.

Fig.5 : Phase diagram of our model for small $u$.

Fig.6 : Unit cells of the six ground states of the square-lattice model.

Fig.7 : Ground-state symmetry of the ferromagnetic six-state clock model.

Fig.8 : Unit cells of the six ground states of the triangular-lattice model:

$U_{i}$ (up-state) has a positive helicity and $D_{i}$ (down-state) a negative helicity.

Fig.9 : The domain wall between $U_{i}$ and $U_{j}$ ground states in the direction 1. Here $i^{\prime}=i+1, i^{\prime \prime}=i-1, j^{\prime}=j+1, j^{\prime \prime}=j-1$.

Fig.10 : Ground-state symmetry of the triangular-lattice model.

Fig.11 : Domain walls (dashed lines) on a distorted triangular lattice at $u=0$. The thick bonds are unhappy (ferromagnetic) bonds which domain walls go across. Notice that there are no unhappy bonds where two domain walls cross.

Fig.12 : Six vertex types of domain walls (dashed lines) with unnormalized Boltzmann factors. Solid lines represent no domain walls. 
Fig.13 : One of the infinitely many ground states for the model with mixedtype interactions.

Fig.14 : Phase diagram of the antiferromagnetic three-state Potts model on a triangular lattice. 